\title{
ABORDAGEM DO CONCEITO DE LIMITE APRESENTADO POR JAMES STEWART E SUAS CONTRIBUIÇÕES PARA A APRENDIZAGEM E FORMAÇÃO DE PROFESSORES DE MATEMÁTICA
}

\section{APPROACH TO THE CONCEPT OF LIMIT PRESENTED BY JAMES STEWART AND HIS CONTRIBUTIONS TO THE LEARNING AND TRAINING OF MATHEMATICS TEACHERS}

\author{
João Nunes de Araujo Neto ${ }^{1}$; Francisco José de Lima ${ }^{2}$; Damião Evandro Barbosa de \\ Sousa ${ }^{3}$
}

\begin{abstract}
RESUMO
No âmbito da formação inicial de professores, as matrizes curriculares de cursos de licenciatura em matemática são constituídas, predominantemente, por cálculo diferencial e integral em diferentes aspectos e o livro didático assume um papel de destaque na aula de cálculo. Esse trabalho tem por objetivo refletir sobre a abordagem de James Stewart na exposição e construção do conceito de limite, considerando aspectos da tendência em educação matemática, resolução de problemas. Em cursos de licenciatura é importante considerar o ensino de limite norteado pelas concepções humanísticas de formação de professores. O estudo mostra-se relevante, por permitir refletir sobre abordagens e pressupostos relacionados ao conceito de limite apresentados por James Stewart no livro Cálculo Volume I, observando suas contribuições para a formação de professores de matemática. De natureza qualitativa e abordagem exploratória, o estudo realizou observações e descrição do conceito de limite apresentado no livro estudado. Destaca-se o problema da tangente como uma motivação para iniciar as discussões sobre a teoria de limites, o problema de velocidade e o estudo de limite de funções reais. A partir da abordagem desse conteúdo, foi possível concluir que uma das estratégias utilizadas por James Stewart, teve como base a resolução de problemas, contudo observou-se no texto a presença de elementos que indicam uma preocupação do autor com aspectos pedagógicos, principalmente a indicação de leituras complementares, aplicações de tecnologia elementos históricos e modelagem,
\end{abstract}

\footnotetext{
${ }^{1}$ Doutorado pela Universidade de São Paulo (USP), professor do Instituto Federal de Educação, Ciência e Tecnologia do Ceará (IFCE), Brasil, Ceará, Cedro. Alameda José Quintino, s/n - Prado, Cedro, Ceará, Brasil, 63400-000. Email: joao.nunes@ifce.edu.br

iDORCID iD: https://orcid.org/0000-0002-8981-7599

2 Doutor em Educação pela Universidade Metodista de Piracicaba (UNIMEP). Professor do Instituto Federal de Educação, Ciência e Tecnologia do Ceará (IFCE) campus Cedro. Endereço: Rua 01, s/n, Conjunto Habitacional, Distrito de Várzea da Conceição, Cedro, Ceará, Brasil, CEP: 63400-000. E-mail: franciscojose@ifce.edu.br

iDORCID iD: https://orcid.org/0000-0001-5758-5159

${ }^{3}$ Especialista pela Faculdade de Ciências Administrativas e de Tecnologia (FATEC), Professor da escola Dr. João Gonçalves de Souza, Brasil, Ceará. R. São Sebastião - Mangabeira - Distrito, Lavras da Mangabeira - CE, 63300-000. Email: prof.evandro190297@ hotmail.com

iDORCID iD: https://orcid.org/0000-0002-0964-537X
} 
evidenciando a influência de tendências em educação matemática para a promoção dos processos de ensino e aprendizagem de limite.

Palavras-chave: Formação inicial de professores; conceito de limite; ensino; aprendizagem.

\begin{abstract}
In the context of initial teacher training, the curricular matrices of undergraduate courses in mathematics are constituted, predominantly, by differential and integral calculus in different aspects and the textbook assumes a prominent role in the calculus class. This work aims to reflect on James Stewart's approach in the exhibition and construction of the concept of limit, considering aspects of the trend in mathematics education, problem solving. In undergraduate courses it is important to consider the teaching of a limit based on the humanistic conceptions of teacher training. The study is relevant because it allows reflecting on approaches and assumptions related to the concept of limit presented by James Stewart in the book Calculus Volume I, observing his contributions to the training of mathematics teachers. Of a qualitative nature and exploratory approach, the study made observations and description of the concept of limit presented in the studied book. The problem of tangent is highlighted as a motivation to start discussions about the theory of limits, the speed problem and the study of limit of real functions. From the approach of this content, it was possible to conclude that one of the strategies used by James Stewart was based on problem solving, however it was observed in the text the presence of elements that indicate a concern of the author with pedagogical aspects, mainly the indication of complementary readings, applications of technology historical elements and modeling, evidencing the influence of trends in mathematical education for the promotion of teaching processes and learning limits.
\end{abstract}

Keywords: Initial teacher training; concept of limit; teaching; apprenticeship. 


\section{Introdução}

No decorrer da história, discussões acerca da matemática e seu ensino vêm sendo evidenciadas no meio acadêmico e social, devido ao caráter humano formativo e suas contribuições para o desenvolvimento tecnológico. Tendo em vista as importantes descobertas realizadas no campo do ensino de matemática é fundamental estabelecer um diálogo entre os conteúdos específicos e as contribuições produzidas pela educação matemática, promovendo uma aproximação reflexiva na tentativa de diminuir a dualidade entre conteúdo e prática pedagógica.

No contexto da formação inicial de professores, as matrizes curriculares das licenciaturas em matemática são constituídas predominantemente por cálculo diferencial e integral em seus mais variados aspectos. Esse pressuposto tem marcado a formação de licenciandos com suas características analíticas, cujo viés curricular mostra a relevância intrínseca do processo de ensino e aprendizagem de cálculo para a formação de professores, ou demonstra um equívoco na construção das matrizes pela importância desproporcional dada a tal conteúdo.

Tendo em vista a importância do cálculo, em particular o conteúdo de limite para formação de futuros professores, o qual traz consigo aspectos intuitivos importantes, possibilitando a formalização da ideia de aproximação à medida que introduz notação matemática e aplicações relacionando a matemática ao cotidiano. Além disso, limite de sequências e funções é um conteúdo que está presente na educação básica, técnica, tecnológica e ensino superior.

É importante considerar a existência de especificidades entre propostas formativas de cursos de bacharelado e licenciatura em matemática. Nas licenciaturas é importante considerar o ensino de limite norteado pelas concepções humanísticas decorrentes do viés pedagógico da formação de professores. Com isso, propõe-se neste trabalho um estudo qualitativo sobre a importância de uma abordagem aplicada e reflexiva para o ensino de limites, considerando o referencial teórico produzido por James Stewart em seu livro Cálculo Volume I, $7^{\circ}$ edição, a partir do contexto da resolução de problemas.

No contexto da formação inicial docente, o livro didático assume um papel importante na aula de cálculo. Nesse sentido, cabe ao docente buscar formas para a exposição de conteúdos, intermediando a relação aluno-conteúdo-aprendizagem. Para 
Santos e Martins (2011, p.10), “os livros trazem ao professor o que deve ser ensinado e como deve ser desenvolvido cada conteúdo em sala de aula, apresentando ainda os resultados que devem ser alcançados". Nesse contexto, é importante observar a forma como o livro apresenta o conceito de limite, verificando especificamente como a tendência em educação matemática Resolução de Problemas pode contribuir para o ensino e a aprendizagem do conteúdo.

Esse estudo mostra-se relevante por possibilitar reverberações sobre abordagens e pressupostos relacionados ao conceito de limite, apresentados por James Stewart no livro Cálculo Volume I, observando suas contribuições para a formação de professores no contexto do curso de Licenciatura em Matemática. Assim, o presente trabalho tem por objetivo refletir sobre a abordagem de James Stewart na exposição e construção do conceito de limite, considerando aspectos da Resolução de Problemas como uma tendência em educação matemática.

\section{Percurso Metodológico}

Esta pesquisa, de natureza qualitativa e abordagem exploratória, pautou-se na realização de observação e descrição do conceito de limite apresentado no livro Cálculo volume I de James Stewart. Conforme Praça (2015), métodos qualitativos apresentam uma relação entre os objetos e os resultados, que não são explicados através de números, mas por interpretação de fenômenos analisados intuitivamente, constituindo uma pesquisa descritiva. Com isso, pode-se dizer que a pesquisa qualitativa realiza-se normalmente em fatos e objetos já estudados.

No contexto da formação de professores, o Livro Cálculo Volume I é utilizado como referencial básico no curso de licenciatura onde os autores atuam. Os aspectos pedagógicos do livro têm despertado o interesse para sua utilização em práticas e pesquisas em grupos de estudos. A ênfase dada às aplicações e resolução de problemas evidenciam seu papel no cotidiano acadêmico dos licenciandos, a medida que motiva diálogos sobre a importância da Matemática para a formação humana.

Diante da problemática deste trabalho, fez-se necessário uma investigação sobre o tratamento dado por James Stewart durante a exposição e construção do conceito de limite. Para tanto, iniciou-se a realização de leituras, resolução de exemplos e problemas 
propostos, objetivando uma organização/sistematização do pensamento do autor sobre como deve ser abordada essa temática. Após esse momento, houve a necessidade de descrever alguns elementos que possibilitassem construir um elo entre o referencial teórico e os objetivos da pesquisa, para delinear uma discussão entre a proposta de James Stewart com os processos de ensino e aprendizagem de limite.

Dentre as leituras foi oportuno conhecer quem foi o autor. James Drewry Stewart, nasceu em 29 de março de 1941 no Canadá, professor emérito de Matemática da Universidade McMaster do Canadá, fez mestrado pela Universidade Stanford e doutorado pela Universidade de Toronto com ênfase em análise harmônica e funcional (WATSON, 2014). Stewart obteve reconhecimento pela produção de uma série de livros didáticos para os ensinos médio e superior. Dentre esses, os livros que mais se destacaram foram direcionados aos cursos de engenharia, física e matemática.

No primeiro capítulo o autor apresenta um resumo dos conteúdos de Álgebra Básica, Geometria Analítica, Funções e Trigonometria, considerando os aspectos: verbal, numérico, visual e algébrico. No segundo capítulo são expostos os conceitos de limite e derivada, onde o autor propõe a discussão de dois problemas motivadores, o problema da tangente e um problema envolvendo conceito físico de velocidade. Com isso, o estudo de limites é mostrado por meio de quatro aspectos: descritivo, gráfico, numérico e algébrico.

Na seção seguinte, serão abordados alguns apontamentos considerados na leitura do livro Cálculo volume I de James Stewart, com a intenção de elencar as contribuições apresentadas pelo autor.

\section{O problema da tangente: Uma motivação para o iniciar as discussões sobre a teoria} de limites

Objetivando iniciar as discussões sobre a teoria de limite sem fazer uso do formalismo matemático abstrato, inicialmente o autor procura mostrar uma noção intuitiva de limite, para tanto, ele entende que é importante deixar claro o significado termo tangente para o cálculo diferencial, observe Figura 1, (a) e (b). 
Figura 1 - Reta tangente ao círculo (a) e reta tangente a curva C (b)

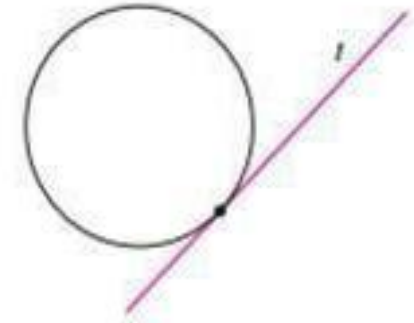

(a)

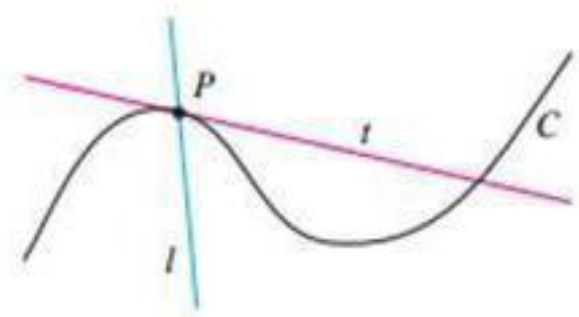

(b)

Fonte: Stewart, 2013.

Em relação a Figura 1, o autor procura mostrar duas concepções historicamente desenvolvidas sobre o termo tangente, à parte (a) faz referência ao livro III da obra Elementos, escrita por Euclides por volta 300 a.C., onde é introduzido o conceito de tangência utilizado na educação básica e superior durante os estudos de geometria plana. A parte (b) da Figura 1, a definição de Euclides não se aplica, uma vez que a reta t toca a curva c em dois pontos, sendo assim uma secante.

A análise de tangência de uma reta em relação a uma curva é um problema um pouco complexo. Observa-se na Figura (b) duas retas 1 e t tocando em um ponto $\mathrm{P}$, sendo $P$ ponto da curva $C$, note que a reta 1 por sua vez intersecta $C$ somente em um ponto, mas não é o tipo de tangência usual utilizada no cálculo. A reta $\mathrm{t}$ apresenta no ponto $\mathrm{P} a$ situação que a teoria de cálculo aborda usualmente, mesmo tocando duas vezes a curva, evidenciando a necessidade do desenvolvimento de uma teoria que formalize ideia de aproximação, possibilitando uma análise "local" do comportamento das curvas e gráficos de funções.

Constata-se a preocupação em mostrar para os alunos a ideia intuitiva de limite utilizando conhecimentos já adquiridos por eles no ensino médio. Neste momento, o livro auxilia o docente a introduzir o contexto histórico e problemas do ensino médio que envolvem essa temática, procurando enriquecer o diálogo formativo acerca da ideia de limite no cálculo diferencial.

No exemplo a seguir, o autor procurou mostrar que é possível determinar a inclinação da reta tangente a uma curva fazendo a aproximação a partir de uma reta secante a curva, possibilitando ao estudante entender matematicamente a ideia de 
aproximação e o conceito de limite. Exemplo: Encontre uma equação da reta tangente à parábola $\mathrm{y}=\mathrm{x}^{2}$ no ponto $(1,1)$.

Figura 2 - Gráfico parábola $\mathrm{y}=\mathrm{x}^{2}$

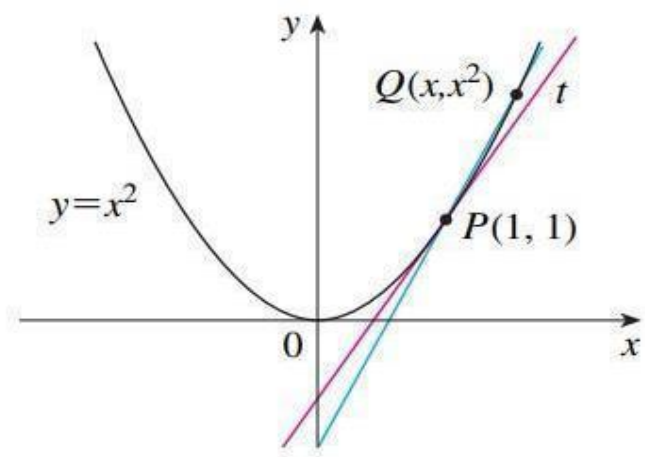

Fonte: Stewart, 2013.

Com o intuito de solucionar essa questão é necessário relembrar o conceito de geometria analítica (inclinação) para exibir a equação da reta tangente t à parábola $y=x^{2}$. O livro ao trazer esse problema clássico do cálculo, encontrar a equação da reta tangente a uma curva em um ponto específico, preparar os discentes para problemas que posteriormente serão resolvidos usando técnicas de derivação. Um ponto positivo para essa abordagem é aproximar os conhecimentos dos alunos com a teoria que será estudada durante o andamento do curso de cálculo I.

Pode-se notar na Figura 2, que são necessários dois pontos para calcular a inclinação da reta secante a curva, observa-se que escolhendo um ponto $Q\left(x, x^{2}\right)$ que está contido na parábola, obtém-se uma aproximação de m fazendo o valor de $x$ aproxima-se de 1, como visto na Figura 2. Calculando a inclinação $m_{P Q}$ da reta secante PQ, usando o fato de que $x \neq 1$ e $P \neq Q, \log m_{P Q}=\frac{x^{2}-1}{x-1}$. Considere a tabela que relaciona valores de $\mathrm{x}$ a valores de $m_{P Q}$, na segunda linha da tabela tem-se $x=1,5$ e $Q\left(x, x^{2}\right)=Q(1,5,2,25)$, decorre a partir da variação de x pela direita e esquerda do número 1 tem-se: 
Tabela 1 - Comportamento de $m_{P Q}$

\begin{tabular}{|l|l|l|l|}
\hline $\mathrm{X}$ & $m_{P Q}$ & $\mathrm{x}$ & $m_{P Q}$ \\
\hline 2 & 3 & 0 & 1 \\
\hline 1,5 & 2,5 & 0,5 & 1,5 \\
\hline 1,1 & 2,1 & 0,9 & 1,9 \\
\hline 1,01 & 2,01 & 0,99 & 1,99 \\
\hline 1,001 & 2,001 & 0,999 & 1,999 \\
\hline
\end{tabular}

Fonte: Stewart (2013)

A tabela 1 mostra que a medida em que $x$ se aproxima de 1, mais próximo Q estará de P. Com isso, $m_{P Q}$ se aproxima de 2 , logo a inclinação da reta tangente $t$ deve ser $m=2$ e portanto observa-se que a inclinação reta tangente é um "limite" das inclinações das retas secantes.

O termo limite aparece no desenvolvimento do texto inicialmente para familiarizar o leitor com a ideia intuitiva que possui, dando sentido à expressão. Associar a palavra limite a um exemplo que traz para o ensino o real significado matemático do conceito, ao introduzir a notação "lim" o autor procura explicar a ideia de aproximação expostos na tabela 1, definindo $\lim _{Q \rightarrow P} m_{P Q}=m$ significando que a inclinação da reta $m_{P Q}$ é $m$ quando Q fica suficientemente próximo de $\mathrm{P}$, outra forma de expressar essa sentença é $\lim _{x} \rightarrow_{1}$ $\frac{x^{2}-1}{x-1}=2$.

Entende-se que a inclinação está tendendo a 2 quando $\mathrm{x}$ tende a $1, \operatorname{logo}$ a inclinação é igual a 2. Usando a forma do coeficiente angular que diz $y-y_{P}=m\left(x-x_{P}\right)$, escreve-se a equação da reta tangente no ponto $P(1,1)$ da seguinte forma: $y-1=2(x-1)$ $\Leftrightarrow y=2 x-1$. A figura seguinte apresenta o comportamento da secante ao gráfico quando Q se aproxima de $\mathrm{P}$. 
Figura 3 - Reta secante tendendo a reta tangente
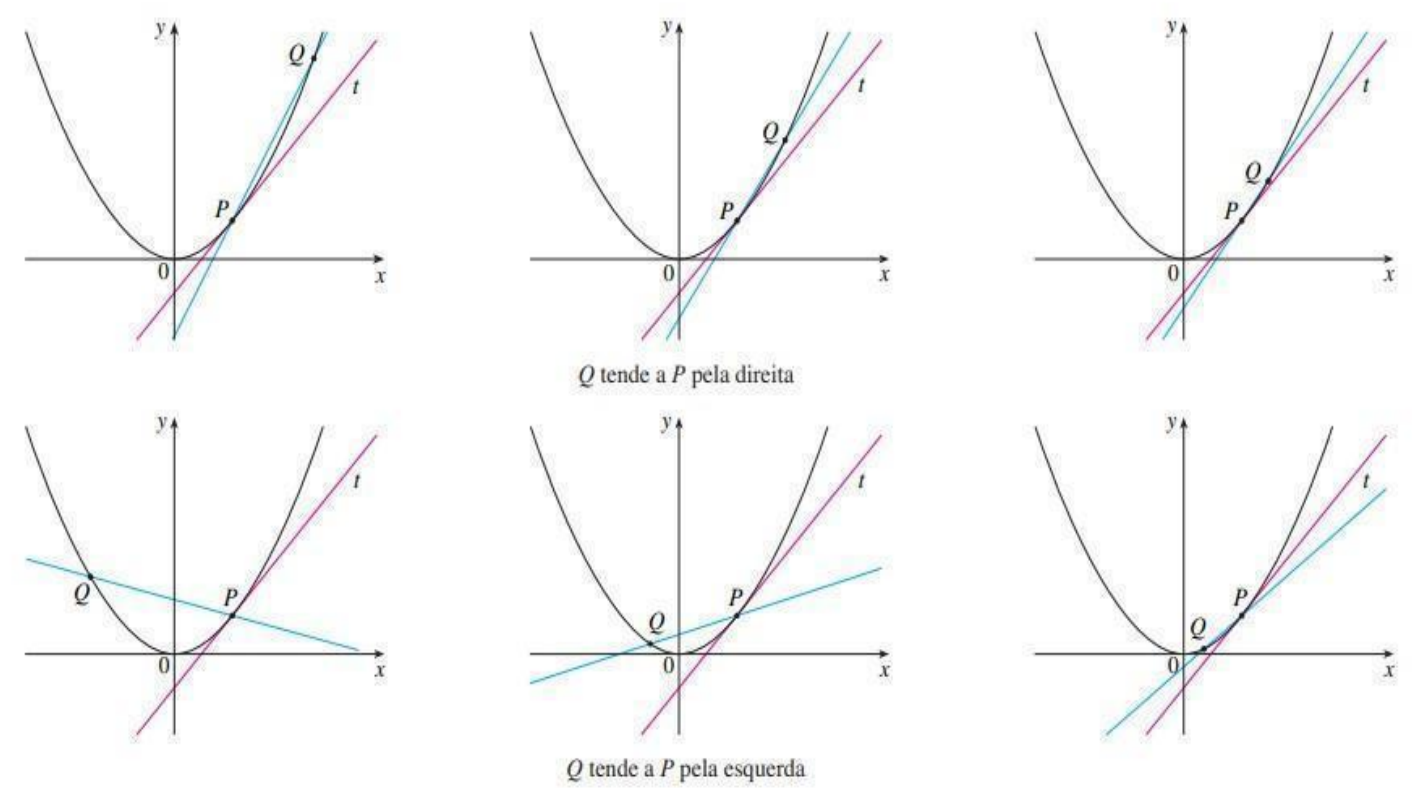

Fonte: Stewart, 2013.

\section{Problema de Velocidade}

Na busca por formalizar o conceito de limite, o autor considera uma situação do cotidiano, cita o caso de um velocímetro em um carro no tráfego urbano, analisando a variação do ponteiro durante o deslocamento do carro, para exemplificar a ideia de velocidade constante e motivar o questionamento a respeito do que seria velocidade instantânea. O exemplo seguinte é uma situação problema que envolve um objeto em queda livre e sua velocidade instantânea. Suponha que uma bola seja solta a partir do ponto de observação no alto da Torre $\mathrm{CN}$, em Toronto, $450 \mathrm{~m}$ acima do solo. Encontre a velocidade da bola após 5 segundos

Stewart (2013, p.78) procura fazer referência ao clássico experimento de Galileu que afirma que a distância percorrida por qualquer objeto em queda livre é proporcional ao quadrado do tempo de queda, no experimento apresentado desprezou-se a resistência do ar, levando isso em consideração, temos que um objeto ao percorremos uma distância em $\mathrm{t}$ segundos e chamamos $s(t)$ em metros a medida segundo a lei de Galileu pode ser expressa dessa forma $s(t)=4,9 t^{2}$. O conceito físico de velocidade média (V)

$$
V=\frac{\Delta s}{\Delta t}
$$


A mudança de posição é expressa em função do tempo pela lei de galileu $s(t)$, a variação de posição é dada $s\left(t_{2}\right)-s\left(t_{1}\right)$ e o tempo decorrido é dado por $t_{2}-t_{1}$. Como o exemplo pede a velocidade instantânea em $t=5$ segundos, é natural analisar a velocidade média considerando uma pequena variação de tempo, $t_{2}=5,1$ e $t_{1}=5$.

$\frac{s\left(t_{2}\right)-s\left(t_{1}\right)}{0,1}=\frac{s(5,1)-s(5)}{0,1}=\frac{4,9(5,1)^{2}-4,9(5)}{0,1}=49,9 \mathrm{~m} / \mathrm{s}$. Esse comportamento expressado na equação pode ser observado na tabela seguinte.

Tabela 2 - Velocidade média com t no intervalo [5,6]

\begin{tabular}{|c|c|}
\hline Intervalo do Tempo & Velocidade média $(\mathrm{m} / \mathrm{s})$ \\
\hline $5 \leq t \leq 6$ & 53,9 \\
\hline $5 \leq t \leq 5,1$ & 49,49 \\
\hline $5 \leq t \leq 5,05$ & 49,245 \\
\hline $5 \leq t \leq 5,01$ & 49,049 \\
\hline $5 \leq t \leq 5,001$ & 49,0049 \\
\hline
\end{tabular}

Fonte: Stewart, 2013.

Como visto na tabela 2, a medida em que a amplitude dos intervalos diminui, se aproxima de 5 a equação vai gerando valores próximo a $49 \mathrm{~m} / \mathrm{s}$. Assim, pode-se estimar o valor da velocidade média em períodos de tempo cada vez menores, começando em $t=$ 5. Portanto a velocidade instantânea é $v=49 \mathrm{~m} / \mathrm{s}$, após 5 segundos.

Nesse sentido, o autor relacionou o problema da tangente com o exemplo da reta tangente a curva, esboçando o gráfico da função. Notou-se que a partir de uma escolha de pontos arbitrário, o deslocamento da bola pode ser observado pelo comportamento do ponto $Q$, à medida que o valor de h diminui o ponto $Q\left(a+h ; 4,9(a+h)^{2}\right)$ aproxima-se do ponto $P\left(a ; 4,9 a^{2}\right)$, como pode ser observado na Figura 5 . 
Figura 4 - Inclinação das retas secante e tangente a parábola
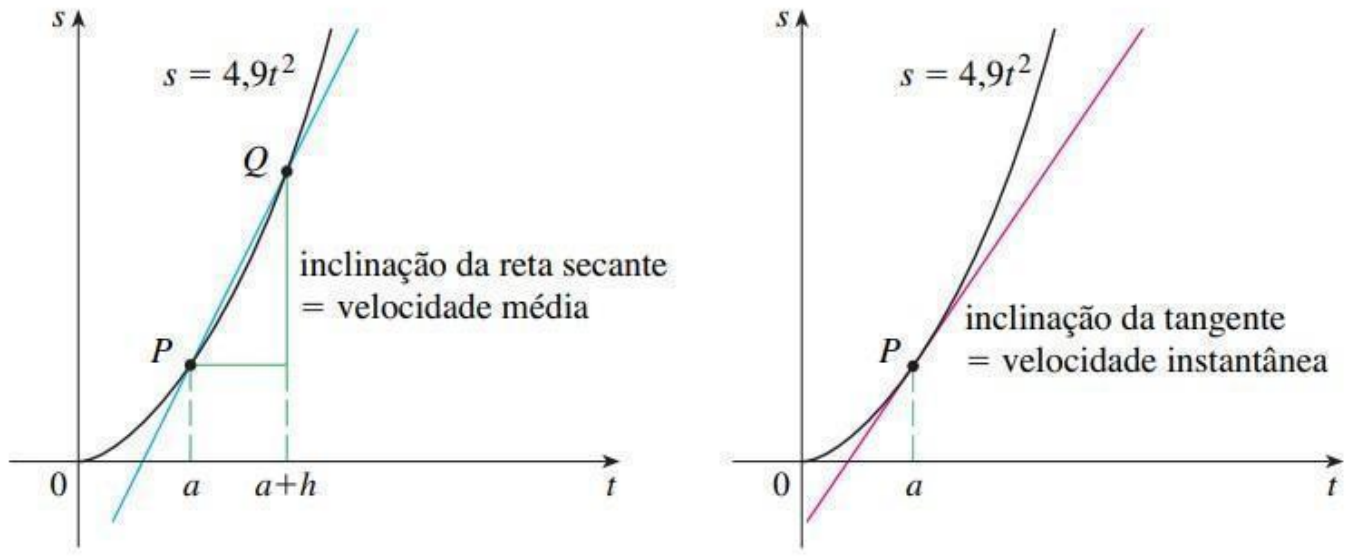

Fonte: Stewart, 2013.

Conclui-se que a inclinação da reta secante é dada $\mathrm{m}_{\mathrm{PQ}}$ representa a velocidade média, quando h tende a 0 , observe que $m_{P Q}$ é a inclinação da reta secante que passa por $P$ e $Q$, e $m$ é o coeficiente angular da reta tangente no ponto $P$.

\section{Estudo de limite de funções reais}

Nessa etapa, o autor propõe com um exemplo definir limite em funções reais. Inicialmente, apresenta um caso particular de uma função polinomial do segundo grau $f(x)=x^{2}-x+2$ e procurar fazer argumentos semelhantes aos exemplos anteriores, veja a tabela seguinte

Tabela 3 - Comportamento de $f(x)$ no intervalo [1,2]

\begin{tabular}{|c|c|c|c|}
\hline $\mathrm{X}$ & $f(x)$ & $\mathrm{x}$ & $f(x)$ \\
\hline 1,0 & 2,000000 & 3,0 & 8,000000 \\
\hline 1,5 & 2,750000 & 2,5 & 5,750000 \\
\hline 1,8 & 3,440000 & 2,2 & 4,640000 \\
\hline 1,9 & 3,710000 & 2,1 & 4,310000 \\
\hline 1,95 & 3,852500 & 2,05 & 4,152500 \\
\hline 1,99 & 3,970100 & 2,01 & 4,030100 \\
\hline 1,995 & 3,985025 & 2,005 & 4,015025 \\
\hline 1,999 & 3,997001 & 2,001 & 4,003001 \\
\hline
\end{tabular}

Fonte: Stewart, 2013.

Note que na tabela $\mathrm{x}$ assume valores próximo a 2 e $f(x)=x^{2}-x+2$ gera valores próximo a 4 e isso também pode ser observado no gráfico da figura 6 . 
Figura 5 - Gráfico de uma função $y=x^{2}-x=2$, com $\mathrm{x}$ tendendo a 2

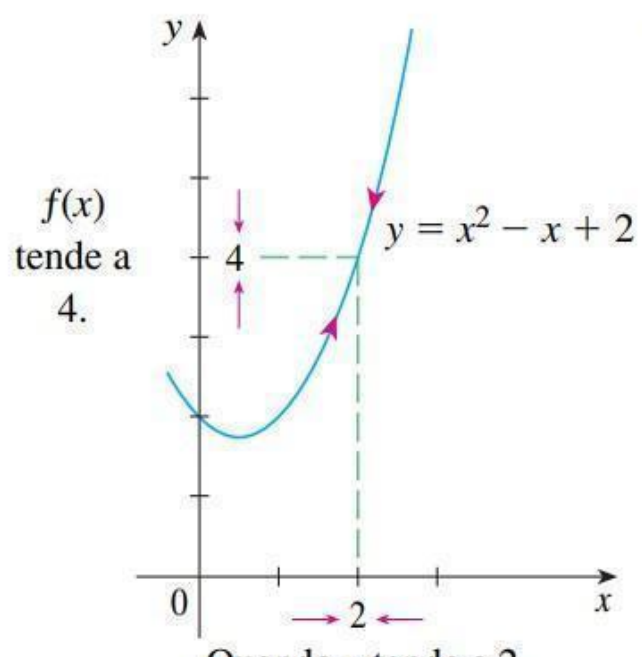

Quando $x$ tende a 2,

Fonte: Stewart, 2013.

O conceito é construído de forma progressiva, o que pode ser observado pela organização dos problemas e exemplos. Foram apresentados problemas com o intuito de naturalizar o leitor com a notação matemática à medida que a teoria é desenvolvida.

A partir desse momento, o autor procura formalizar o conceito do limite, apresentando a seguinte expressão $x \rightarrow 2\left(x^{2}-x+2\right)=4$, essa pode ser lida da seguinte forma "o limite da função $x^{2}-x+2$ quando x tende a 2 é igual a 4. Após o desenvolvimento de uma base intuitiva, o autor usa uma definição na tentativa de explicar a forma de ler a notação de limite na presença da notação de função, mostrando a transição gradual entre os exemplos aplicados e o conceito de limite, na busca de preparar o leitor para uma definição formal.

Definição: Suponha que f(x) seja definido quando está próximo ao número a. (Isso significa que fé definido em algum intervalo aberto que contenha a, exceto possivelmente no próprio a.) Então escrevemos $f(x)=L$. Logo, o limite da função quando x tende a é igual a $\mathrm{L}$.

Nota-se que se tomarmos valores arbitrários para a função f(x), ela se aproximará de $\mathrm{L}$, tornando os valores de $\mathrm{x}$ cada vez mais próximos de a. Mas nunca $\mathrm{x}=\mathrm{a}$ ou seja $\mathrm{x}$ $\neq \mathrm{a}$. 
Figura 6 - Gráficos de casos de funções que são ou não limites

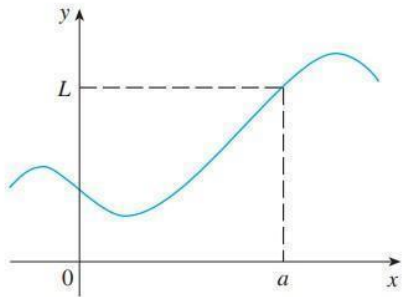

(a)

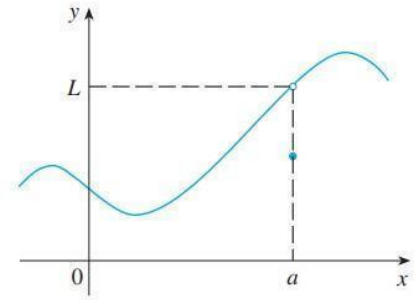

(b)

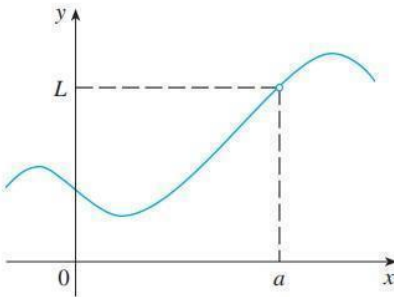

(c)

Fonte: Stewart, 2013.

Na Figura 6, o autor procura analisar com base na definição anterior, três possíveis casos que expressam a relação entre uma função e um ponto. caso (a) a função está definida no ponto e além disso $f(a)=L$ (mas no estudo de limites essa informação não possui relevância), no caso (b) a função está definida em a, mas $f(a) 6=L$ e no caso (c) a função não está definida em a. Em todos os casos o limite da função é igual a $L$ quando x está próximo a.

O exemplo a seguir aborda uma característica mais técnica do conceito, exigindo do docente uma mediação mais cuidadosa para evitar problemas de interpretação, gerando dificuldades no aprendizado dos demais conceitos do cálculo como a derivada e integral. Exemplo: Estime o valor de $\frac{x-1}{x^{2}-1}$

Na construção da solução deste exemplo o autor mostra de maneira analítica e geométrica a forma de se entender o problema, para deixar claro sua intenção de estudar o comportamento da função quando x se aproxima de 1. Utilizando-se valores próximos de 1 na função $f(x)=\frac{x-1}{x^{2}-1}$, tanto pela direita ou pela esquerda, mas sempre $x \neq 1$. A tabela ilustra o comportamento da função.

Tabela 4 - Comportamento de $f(x)$

\begin{tabular}{|c|c|c|c|}
\hline $\mathrm{x}<1$ & $f(x)$ & $\mathrm{x}>1$ & $f(x)$ \\
\hline 0,5 & 0,666667 & 1,5 & 0,400000 \\
\hline 0,9 & 0,526316 & 1,1 & 0,476190 \\
\hline 0,99 & 0,502513 & 1,01 & 0,497512 \\
\hline 0,999 & 0,500250 & 1,001 & 0,499750 \\
\hline 0,9999 & 0,500025 & 1,0001 & 0,499975 \\
\hline
\end{tabular}

Fonte: Stewart, 2013. 
Observando a tabela, têm-se que para $x<1$ ou $x>1$ a função $f(x)$ aproximou-se de 0,5 . Portanto, $\frac{x-1}{x^{2}-1}=0,5$. O gráfico da função $y$, a qual não está definida para $x=1$, tipo o caso (c) Figura 6, é observado na Figura 7.

Figura 7 - Comportamento do gráfico da função $y=f(x)$.

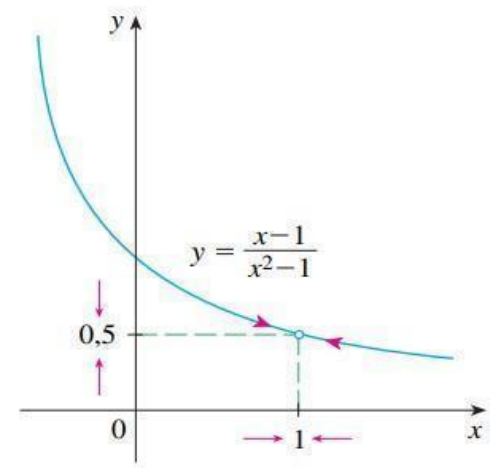

Fonte: Stewart, 2013.

Na situação seguinte o autor apresenta um exemplo tipo um caso (b) da Figura 7 $g(x)=\frac{x-1}{x-1}$, se $x \neq 1$ e $g(x)=2$, se $x=2$

O autor propõe ao estudante observar que mesmo função tenha seu valor definido em outra imagem quando $x=1$, o limite da função não muda em relação ao exemplo anterior.

Figura 8 - Gráfico do comportamento de uma função quando x tende 1

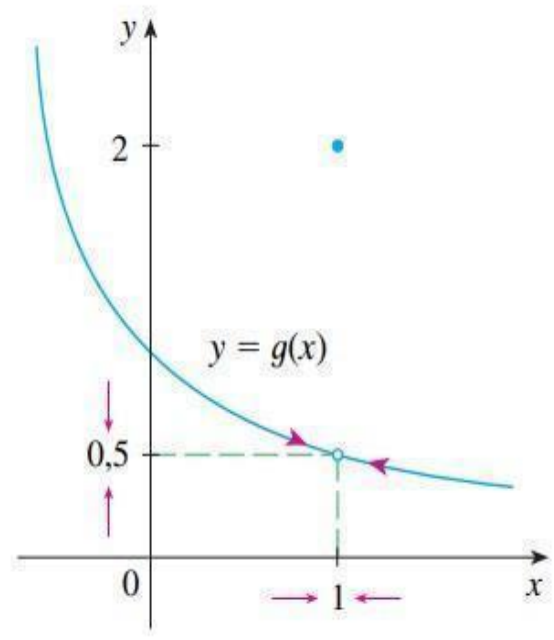

Fonte: Stewart, 2013. 


\section{Considerações Finais}

Com relação à temática desse trabalho, o qual foi desenvolvido considerando dois tópicos presentes na licenciatura em matemática, (estudo de limite e resolução de problemas), os quais pela sua importância chegam a dar um estereótipo para o professor de matemática, no sentido de que os docentes são reconhecidos por resolver problemas.

Revisitando a temática abordada, podem-se destacar dois pontos fundamentais que nortearam os estudos, o primeiro foi observar a importância da Educação Matemática e da Resolução de Problemas na formação de professores para o ensino de matemática. O segundo, reconhecer o papel do Livro e sua influência nos processos de ensino e aprendizagem, pois a forma de abordagem escrita dos autores podem implicar diretamente na construção do conhecimento matemático em sala de aula.

Para refletir sobre possíveis contribuições para a formação inicial de professores de matemática, a literatura estudada indica que o livro contribui à medida que apresenta os conceitos matemáticos de forma aplicada, diminuindo a abstração inerente à linguagem simbólica. Também propõe intervenções para aproximar o conteúdo científico com o conhecimento prévio dos alunos, além de apresentar repertório de exemplos e exercícios, podendo contribuir para o planejamento do professor.

As considerações feitas durante a pesquisa mostraram-se relevantes, no sentido de enfatizar a importância de refletir sobre o livro didático para a formação docente. Durante o desenvolvimento da exposição descritiva do conceito de limite, foi possível concluir que uma das estratégias utilizadas pelo autor teve como base a resolução de problemas, contudo observamos no texto a presença de elementos que indicam uma preocupação do autor com aspectos pedagógicos, por exemplo, a indicação de leituras complementares, aplicações de tecnologia, elementos históricos e modelagem, evidenciando a influência de tendências em educação matemática.

Enfim, essa pesquisa trata sobre conceitos específicos da formação de professores de matemática, com o intuito de contribuir com o debate sobre o ensino de cálculo e formação de professores. Tais assuntos foram abordados de forma sintética para subsidiar as discussões e fundamentar os questionamentos sem prejudicar a clareza e objetividade do texto. 


\section{Referências}

LOPES, Jairo de Araujo. Livro didático de matemática: concepção, seleção e possibilidades frente a descritores de análise e tendências em educação matemática. 2000. 264 p. Tese (doutorado) - Universidade Estadual de Campinas. Faculdade de Educação, Campinas, SP. Disponível em: <http://www.repositorio.unicamp.br/ handle/REPOSIP/253415>. Acesso em: 11 junho 2020.

PRAÇA, F. S. G. Metodologia da pesquisa científica: organização estrutural e os desafios para redigir o trabalho de conclusão. Revista Eletrônica Diálogos Acadêmicos, v. 8, n. 1, p. 72-87, 2015. Disponível em: http://www.uniesp. edu.br/sites/_biblioteca/revistas/20170627112856.pdf. Acesso em: 11 junho 2020.

STEWART, J. Cálculo - Volume I. Tradução da $7^{\text {a }}$ Edição Norte-Americana. 7. ed. São Paulo: Cengage Learning Edições Ltda, 2013.

SANTOS, Vanessa dos Anjos.; MARTINS, Liziane. A importância do livro didático. Candombá-Revista Virtual, v. 7, n. 1, p. 20-33, 2011. Disponível em: http://revistas.unijorge.edu.br/Portal/index.php/Candomba/about/pdf. Acesso em: 11 junho 2020.

WATSON, HG. As muitas partes de James Stewart. Revista DailyXtra - Publicado em 10 de dezembro de 2014. Disponível em: https://web.archive.org/web/2014121323

2150/http://dailyxtra.com/toronto/life/proud-lives/the-many-parts-james-stewart-96867. Acesso em: 12 jan. 2021. 